\title{
An Empirical Study on the Mechanism of Financial Support to the Development of Cultural Industry in Guizhou County
}

\author{
Huafeng Chen 1, 2, 3, a , Mu Zhang 1, b, * \\ ${ }^{1}$ School of Finance, Guizhou University of Finance and Economics, Guiyang Guizhou 550025, \\ China \\ ${ }^{2}$ Guizhou Institute for Urban Economics and Development, Guizhou University of Finance and \\ Economics, Guiyang Guizhou 550025, China \\ ${ }^{3}$ Guizhou Institution for Technology Innovation \& Entrepreneurship Investment, Guizhou University \\ of Finance and Economics, Guiyang Guizhou 550025, China \\ a2272364739@qq.com, brim_007@163.com \\ ${ }^{*}$ Corresponding author
}

Keywords: financial scale, financial efficiency, financial structure, multiple linear regression, cultural industry, financial support.

Abstract. Based on the data of 88 districts and counties of Guizhou Province, this paper takes the financial scale, financial efficiency, financial structure and the number of cultural industry practitioners to reflect the situation of financial support. A multiple linear regression model was established to analyze the current situation of financial support cultural industry development in Guizhou province. The study found that financial scale, financial structure and the number of cultural industry practitioners have a significant impact on the value of the cultural industry, and the financial efficiency has no significant impact on the added value of the cultural industry. The empirical results show that financial support has positive impact on the development of cultural industry in Guizhou.

\section{Introduction}

The development of cultural industry in our country is relatively late compared with the developed countries, but the development of the cultural industry is very fast. According to the date of the added value released by National Bureau of statistics in 30 August 2015 on 2016 national culture industries and related industries, combined with the first half of 2016 related data, the increase value of national culture industries and related industries was 27235 yuan in 2015, compared with last year nominal growth of $11 \%$ over the same period, which is 4.6 percentage points higher than the nominal GDP growth rate in the same period, showed a rapid growth trend. The development of cultural industry in Guizhou province also showed a good momentum. In recent years, the growth rate of the cultural industry in Guizhou Province was $40 \%$, to achieve the added value 29.9 billion yuan. But the cultural industry accounted for a smaller proportion of GDP, about $3 \%$. So the development of cultural industry has a huge space for development. Optimizing the culture industrial structure is playing a more and more important role.

Historical experience tells us that the development of any industry can not be separated from the financial policy and financial policy support. Increasing the financial industry to support the cultural industry and promoting the cultural industry and the financial industry in a comprehensive docking is the objective needs to foster new economic growth point[1]. At present, some domestic scholars analyze the current situation of cultural industry financing, Such as: Lin Lin, Xu Guangping (2010) study and explore measures and methods of the specific implementation of the financial policy to support the cultural industry development[2]. In terms of the cultural industry in Colleges and universities, Guan Wei, Zhao Zehao, Guan Meng (2012) believe that the cultural industry of colleges and universities should undertake the important mission to promote the innovation and development of China's cultural industry[3]. On the financial support for the development of cultural industries facing the problem, Peng Zhongping, Nie Yong (2013) through the research on the status of Guangxi province cultural industry financial support, analysis the existing problems and finally puts forward the 
policy recommendations of financial support for the development of cultural industries[4]. At the same time, Wang Xianming (2011) from the view of financial support for the development of cultural industries faced with the problem, analysis the inherent mechanism of financial support for the development of cultural industries[5]. And, Hou Ying (2016) emphasizes the characteristics of financial innovation and the development stage of cultural enterprises in the financing of cultural industry and explores the financial support system to promote the development of cultural industry innovation path[6].

In summary, the domestic scholars in the financial support for the development of cultural industry has a wealth of research results. But in this respect, the many researches are still qualitative analysis and the empirical analysis is less. This paper will be based on the data of 88 districts and counties of Guizhou province, to establish multiple linear regression model and make an empirical analysis on the status of financial support to cultural industry in Guizhou Province.

\section{Multiple Linear Regression Model of Financial Support for Cultural Industry Development Model Construction}

There maybe exist a complex relationship between objective phenomena. Some of the changes in the number of variables involved in the relationship between the number of variables. Cross-sectional data is the observation value that a group have the same characteristic variable in the same population at the same time[8]. Combined with the availability of data, we select the cultural industry added value (y) for the explained variable, financial scale $\left(\mathrm{x}_{1}\right)$, financial efficiency $\left(\mathrm{x}_{2}\right)$ and financial structure $\left(\mathrm{x}_{3}\right)$ as explanatory variables. According to the Solow model, we assume that the number of employees in the cultural industry at the end of the year $\left(\mathrm{x}_{4}\right)$ is the exogenous control variable of the model.

A multivariate linear regression model was constructed as follows:

$$
y=\alpha_{0}+\alpha_{1} x_{1}+\alpha_{2} x_{2}+\alpha_{3} x_{3}+\alpha_{4} x_{4}+\varepsilon
$$

$\mathrm{y}$ as the explained variable, indicating the added value of the cultural industry (billion);

$\mathrm{x}_{1}$ as the explanatory variable, indicating the financial scale;

$\mathrm{x}_{2}$ as the explanatory variable, indicating financial efficiency;

$\mathrm{x}_{3}$ as the explanatory variable, indicating the financial structure;

$\mathrm{x}_{4}$ as the exogenous control variable, indicating the number of employees in the cultural industry at the end of the year (person);

$\varepsilon$ represents the random perturbation term of the regression equation ;

$\alpha_{0}$ is a constant term, $\alpha_{1}, \alpha_{2}, \mathrm{~K} \alpha_{4}$ represent the coefficients of the variables.

\section{Data Sources and Processing}

As an empirical study of the financial support effect of the county cultural industry development in Guizhou Province, this paper refers to the relevant research results at home and abroad. The financial scale is measured by the balance of RMB loans of financial institutions; the financial efficiency is measured as the ratio of fixed asset investment to individual savings deposits; the financial structure is measured by the ratio of indirect financing to total financing.

Sample interval is set to 2014. The sample data is selected from the 《Guizhou Statistical Yearbook 2015》 and the data of the actual investigation by Guizhou Province People's Political Consultative Conference, and we fill the missing data by empirical estimation.

According to the data, this paper uses SPSS22. 0 software to estimate the parameters of the formula (1).

Step 1: import the Excel data file into the SPSS22. 0 software.

Step 2: Click the menu "Analysis -> Regression Analysis -> Linear Regression" to open the "Linear Regression" dialog box, the variable y is selected into the "dependent variable" list box, as linear 
regression analysis of the explained variables; The variable $\mathrm{x}_{1}, \mathrm{x}_{2}, \mathrm{x}_{3}, \mathrm{x}_{4}$ are selected into the "independent variable" list box as an explanatory variable for linear regression analysis.

Step 3: Click the "Statistics" button to open the "Linear Regression: Statistics" dialog box. In order to select the "simulation goodness of fit", "regression coefficient: estimation" and "a total linear diagnosis" and so on.

Step 4: Click "OK" button to get the results of multiple linear regression.

\section{Empirical Results and Analysis}

After running the SPSS22. 0 software, the results are as follows.

Table 1 Model fitting

\begin{tabular}{|c|c|c|c|c|}
\hline Model & $\mathrm{R}$ & $\mathrm{R}^{2}$ & Adjusted $\mathrm{R}^{2}$ & Standard Estimation Error \\
\hline 1 & 0.841 & 0.707 & 0.693 & 2.36543 \\
\hline
\end{tabular}

Table 1 is the model fitting condition, which reflects the fitting effect of the model. From the above table we can see that the $R$ value is 0.841 , the adjusted $R^{2}$ is 0.693 , the model's fitting degree is higher, the model constructed can be suitable for the current data analysis.

Table 2 Model explanatory variables coefficient significance test

\begin{tabular}{|c|c|c|c|c|c|c|}
\hline \multicolumn{2}{|c|}{ Model } & \multicolumn{2}{|c|}{ Non - Normalized Coefficient } & Normalized Coefficient & \multirow{2}{*}{ Sig. } \\
\cline { 2 - 7 } & $\mathrm{B}$ & Standard Deviation & Beta & & .389 \\
\hline \multirow{3}{*}{1} & Constant & .588 & .678 & & 0.867 & .000 \\
\cline { 2 - 7 } & $\mathrm{x}_{1}$ & .010 & .001 & .720 & 9.395 & .200 \\
\cline { 2 - 7 } & $\mathrm{x}_{2}$ & .325 & .252 & .079 & 1.290 & .026 \\
\cline { 2 - 7 } & $\mathrm{x}_{3}$ & 3.562 & 1.575 & .139 & 2.262 & .052 \\
\cline { 2 - 7 } & $\mathrm{x}_{4}$ & .000 & .000 & .146 & 1.967 & \\
\hline
\end{tabular}

Table 2 is the model variable coefficient significance test. The significant level of the 4 variables in the model is $0,0.200,0.026,0.052$. We have given the significant level of $10 \%, \mathrm{x}_{1}, \mathrm{x}_{3}$, and $\mathrm{x}_{4}$ passed the significance level test. That is, financial scale, financial structure and the number of employees in the cultural industry at the end have a significant impact to the cultural industries added value . Financial efficiency has no significant effect on the added value of cultural industry. So $\mathrm{x}_{2}$ is removed.

After excluding $\mathrm{x}_{2}$, the model changes to

$$
y=\alpha_{0}+\alpha_{1} x_{1}+\alpha_{3} x_{3}+\alpha_{4} x_{4}+\varepsilon
$$

Using SPSS22.0 software to repeat the steps in previous section, the regression results of multiple linear regression models after the elimination of variables are obtained. The results are as follows.

Table 3 Model fit of the excluded variables

\begin{tabular}{|c|c|c|c|c|}
\hline Model & $\mathrm{R}$ & $\mathrm{R}^{2}$ & Adjusted $\mathrm{R}^{2}$ & Standard Estimation Error \\
\hline 1 & .837 & .701 & .691 & 2.37478 \\
\hline
\end{tabular}

Table 3 is a summary of the model after removing $\mathrm{x}_{2}$. It reflects the fitting effect of the adjusted model. From the above table we can see that the $R$ value is 0.840 , the adjusted $R^{2}$ is 0.699 , and the model's fitting degree is high. It is basically consistent with the data of the model summary before the elimination of variables. 
Table 4 The significant test of the variables of the model after the elimination of variables

\begin{tabular}{|c|c|c|c|c|c|c|}
\hline \multicolumn{2}{|c|}{ Model } & \multicolumn{2}{|c|}{ Non - Normalized Coefficient } & Normalized Coefficient & \multirow{2}{*}{ Sig. } \\
\cline { 3 - 7 } & B & Standard Deviation & Beta & & .000 \\
\hline \multirow{3}{*}{1} & Constant & 1.357 & .325 & & 4.170 & .000 \\
\cline { 2 - 7 } & $\mathrm{X} 1$ & .010 & .001 & .705 & 9.270 & .026 \\
\cline { 2 - 7 } & $\mathrm{X} 3$ & 3.580 & 1.581 & .139 & 2.264 & .065 \\
\cline { 2 - 7 } & $\mathrm{X} 4$ & .000 & .000 & .139 & 1.873 & \\
\hline
\end{tabular}

Table 4 shows the significance of the model explanatory variable coefficient after eliminating $\mathrm{x}_{2}$. The significance level of the three variables in the model is $0,0.026,0.065$. In the case of a given significance level of $10 \%$, all three explanatory variables passed the significance level test. That is, financial scale, financial structure and the number of employees in the cultural industry at the end have a significant impact to the cultural industries added value. The coefficients are 0.010, 3.580, 0.000135 and the constant term is 1.357 .

The regression analysis shows that the financial scale, financial structure and the number of employees at the end of the cultural industry are positively correlated with the added value of the cultural industry. Indicating that for each additional unit of financial scale, the added value of cultural industries increased by 0.010 units; For each additional unit of the financial structure, the added value of cultural industries will increase by 3.580 units; The number of cultural industry practitioners at the end of the year increased by 1 units, the cultural industry to increase the value of 0.000135 units.

\section{Conclusions}

The study found that the financial scale, financial structure and the number of cultural industry practitioners have a significant impact on the added value of the cultural industry, and the financial efficiency has no significant impact on the added value of the cultural industry. And the influence of financial structure on the added value of cultural industry is great, the number of employees in the cultural industry at the end of the year has little influence on the added value of the cultural industry. The empirical results show that financial support has positive impact on the development of cultural industry in Guizhou. The development of cultural industry will promote the prosperity of Guizhou's economy. Therefore, the development of cultural industries should be in a priority, especially in areas such as Guizhou has a distinctive local characteristics. Through an empirical study on the development of cultural industry in Guizhou, it is found that financial support is essential to the development of cultural industry.

\section{Acknowledgements}

This work was financially supported by National Natural Science Foundation of China (71263011).

\section{References}

[1] Liu Yuzhu. The present situation and Prospect of the development of financial support cultural industry [J]. China Finance, 2011, (22): 15-17.

[2] Baoke Lin, Xu Guangping. The key points about the financial policy support for cultural industry development and prosperity of the theory of [J]. Theoretic Observation, 2010, (4): 159-160. 
[3] Guan Wei, Zhao Zehao, Guan Meng. Financial support for the development of cultural industry -Based on the perspective of the operation of the university cultural science and Technology Park [J]. Chinese University Science \& Technology, 2012, (3): 69-71.

[4] Peng Zhongping, Nie Yong. Research on financial support for the development of cultural industry in Guangxi [J]. Journal of Guangxi University for Nationalities, 2013, (4): 133-135.

[5] Wang Xianming. Research on the financial support mechanism for promoting the development of cultural industry [J]. Journal of Chinese Academy of Governance, 2011, (6): 68-70.

[6] Hou Ying. Innovation of financial support system for cultural industry [J]. On Economic Problems, 2016, (3): 80-85.

[7] Jin Tingliang. Econometrics [M]. Chengdu: Southwest University of Finance and Economics Press, 2011. 